\title{
Article \\ A Theoretical Modeling Analysis of Adapted Composite CNT Bundle for High-Speed VLSI Interconnect
}

\author{
Abu Bony Amin $1,+, *\left(\mathbb{D}, \mathrm{S}\right.$ M Shakil ${ }^{2}$ and Muhammad Sana Ullah ${ }^{3}$ (]) \\ Affiliation 1; abonyamin5908@floridapoly.edu \\ Affiliation 2; sshakil2152@floridapoly.edu \\ Affiliation 3; msullah@floridapoly.edu \\ * Correspondence: abonyamin5908@floridapoly.edu \\ + Current address: Department of Electrical and Computer Engineering, University of Massachusetts \\ Amherst, MA - 01002 \\ $\ddagger$ These authors contributed equally to this work.
}

\begin{abstract}
The aroused quest to reduce the delay at interconnect level is the main urge of this paper to come across a configuration of Carbon Nanotube (CNT) bundle namely squarely packed bundle of composite CNTs. The approach, demonstrated in this paper, adapts the composite bundle to adopt for high speed Very Large Scale Integration (VLSI) interconnect with technology sizing down. To reduce the delay of the proposed configuration of composite CNT bundle, the behavioral change of Resistance $(R)$, Inductance $(L)$ and Capacitance $(C)$ has been observed with respect to both width of the bundle and diameter of the CNTs in the bundle. Consequently, the performance of the modified bundle configuration is compared with previously developed configuration namely squarely packed bundle of dimorphic MWCNTs in terms of propagation delay and crosstalk delay at local, semiglobal and global level interconnect. The proposed bundle configuration is ultimately enacted as better one for $32 \mathrm{~nm}$ and $16 \mathrm{~nm}$ technology node and suitable for $7 \mathrm{~nm}$ as well.
\end{abstract}

Keywords: Composite CNT bundle; crosstalk delay; interconnect; propagation delay; RLC model.

\section{Introduction}

The overwhelming exploitation of interconnect to the device delay makes researcher to weigh Carbon Nanotubes (CNTs) for the possession pertinent to long mean free path [1], electrical properties [1,2], thermal properties [2,3], electromigration and current density [4]. Moreover, crosstalk delay is potential stymie for CNTs due to capacitive coupling between adjacent bundle [5]. As it has already been anticipated in [1] that the performance will be meliorated with further technology scaling, CNT can provide much better performance with exploration of some features.

It is claimed in $[5,6]$, that mutual inductance doesn't have a considerable impact on crosstalk-induced delay and glitch instead coupling capacitance along with electrostatic and quantun capacitance puts main contribution. It is also noticed from [7] that the graphitized electron beam-induced deposition (EBID) carbon has capability to produce a low-resistance ohmic contact to multiple shells of MWCNT in the context of making high-performance electrical interconnect structure for the next-generation electronic circuits.

While the configuration of the bundle of CNTs is seizing attention, a trade-off between propagation delay and crosstalk delay is conspicuous [8] as it is mentioned in [5] that SWCNT and DWCNT shows poorer performance than $\mathrm{Cu}$ based interconnect owing to higher coupling capacitance. Due to this reason, we endeavored to avoid putting any SWCNT and DWCNT on the edge of the bundle in our configuration.

To improve the crosstalk delay along with propagation delay, some works [6,9-12] are conducted by introducing different bundle configurations with compositing both 
MWCNT and SWCNT in the bundle. Rai et al. claimed that the structure with MWCNTs surrounded by SWCNTs yields better performance by considering the tunneling and intershell coupling between adjacent shells by depicting four different structures in [10]. In [11], the same group in contrast shows the structure, SWCNTs and MWCNTs possessing equal halves vertically, as the best one in terms of frequency noise amplitude by delineating the same four structures. However, the evidence is provided in [6,9] by varying the relative position of MWCNTs and SWCNTs in the bundle that CNTs with spatial distribution, putting the SWCNTs entirely wrapped up by MWCNTs in the bundle, indulges lower crosstalk delay than that with random arrangement. It is also remarkable from [12] that a delay efficient configuration of composite bundle is proposed though the crosstalk delay performance of this configuration isn't well-proved since this work opposes the fact, mentioned in $[6,9]$, that SWCNTs are mounted over boundary of the bundle.

The aim of this paper is to present an innovative diameter controlled configuration to alleviate the propagation delay and crosstalk delay of size shrinking interconnect which is feasible from fabrication aspect. This configuration is presented here with detailed analytical analysis and comparison results with the aim of better investigating its performance and enlightening its advantages. To analyze the delay performance, the analytical delay model has been obtained using the parameters from [13-15].

The residue of this article is going to follow upcoming course. A modified configuration is proposed as the successor of the configuration, introduced in [16], in Section 2. Section 3 is used to develop the mathematical models for RLC elements for isolated CNTs and eventually for composite bundle based on the configuration exposed in Section 2. Section 4 is dedicated to the interest of simulating and analyzing the performance indicators, propagation delay and crosstalk noise for different technology nodes and showing a comparison with the previously well-developed research work [16]. The conclusion including further research direction is ultimately delimited in Section 5.

\section{Modified Composite Bundle Configuration}

Our endeavor in this paper is to enhance the performance by altering the configuration, shown in Fig. 1(a). In this newly introduced configuration, illustrated in Fig. 1(b), the replacement of smaller MWCNTs is taken place with a bunch of SWCNTs which are wrapped up by the larger MWCNTs. This modified approach is virtue of increasing the number of CNTs in the bundle so that we can fill up the unoccupied space with SWCNT more efficiently and densely.

The proposed configuration is going to follow the geometric pattern, similar to the previous configuration from [16,17], to accommodate more number of CNTs in the bundle. In addition to every four larger MWCNTs are forming square by taking the vertices of the square in the center of those MWCNTs, another square forms in the center of the square. A certain number of SWCNTs is accommodated in this newly formed square which will follow the hexagonally packed pattern. The number of larger MWCNTs and the number of SWCNTs in the bundle are calculated using (1).

By considering the one third of the shells of MWCNTs as metallic [18], the average number of conducting channels for a shell can be calculated using (1)

$$
N_{\mathrm{c}}(i) \approx \begin{cases}\alpha T D_{\mathrm{i}}+\beta & \text { if } D_{\mathrm{i}}>\frac{D_{\mathrm{T}}}{T} \\ \frac{2}{3} & \text { if } D_{\mathrm{i}} \leq \frac{D_{\mathrm{T}}}{T}\end{cases}
$$

where, $\alpha=2.04 \times 10^{-4} \mathrm{~nm}^{-1} K^{-1}, \beta=0.425, D_{\mathrm{T}}=1300 \mathrm{~nm} \cdot K$ and $D_{\mathrm{i}}$ is the diameter of the $i^{\text {th }}$ shell of the MWCNT.

For the sake of simplicity of calculation and shows the relation among every parameter, we are going to pursue further by considering a constant ' $a$ ' which is the side of 


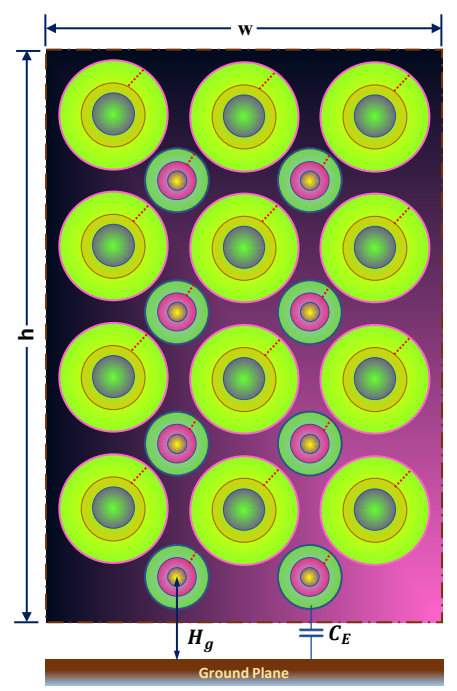

(a)

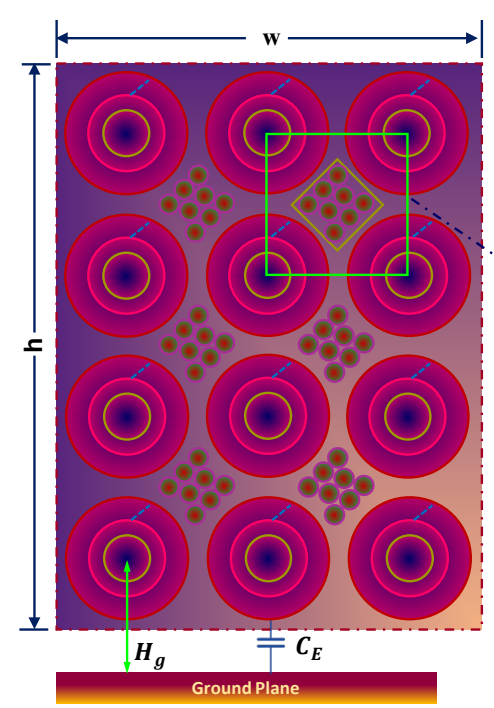

(b)

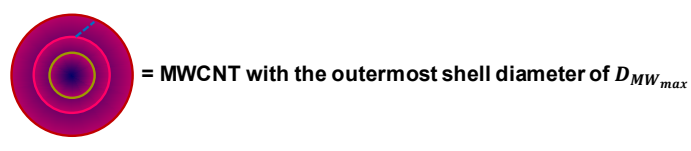

= SWCNT with diameter of $D_{S W}$

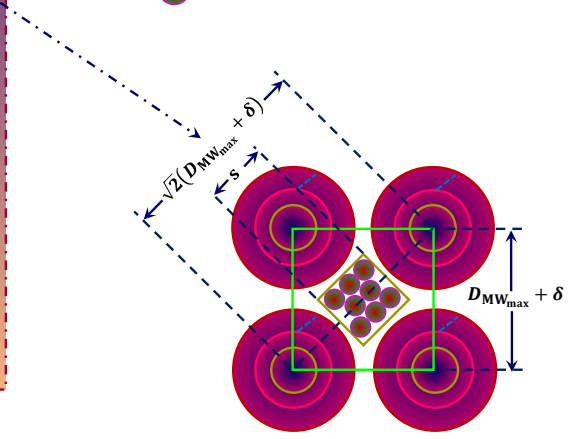

(c)

Figure 1. In both architecture the size of the larger MWCNTs are same. The main modification happened in the introduced configuration by means of smaller MWCNTs replaced by a bunch of SWCNTs with same predefined diameter according to the space available based on the technology nodes. Van der Walls distance of $\delta=3.4 \mathrm{~nm}$ is maintained between any MWCNT and adjacent CNTs.

the square formed by the MWCNTs in Fig. 1. So, the diameter of the outermost shell of MWCNT is

$$
D_{\mathrm{MW}_{\max }}=a-\delta
$$

The number of shells of MWCNTs can be calculated using following formula (3) according to $[9,12,19]$.

$$
n=\left\lceil\frac{D_{\mathrm{MW}_{\max }}-D_{\mathrm{MW}_{\min }}}{2 \delta}\right\rceil
$$

According to the geometry of circle, we know that the diagonal of the bigger square from Fig 1 is $\sqrt{2} a$. Hence, we may calculate the side of the smaller square in (4) from Fig 1

$$
s=\sqrt{2} a-(a+\delta)=(\sqrt{2}-1) a-\delta
$$

To calculate the plausible number of accommodated SWCNTs in the smaller square of Fig. 1,

$$
N_{\mathrm{SW}}=\left(N_{\mathrm{SW}_{\mathrm{H}}} N_{\mathrm{SW}_{\mathrm{V}}}-\left\lfloor\frac{N_{\mathrm{SW}_{\mathrm{V}}}}{2}\right\rfloor\right)
$$

where,

$$
N_{\mathrm{SW}_{\mathrm{H}}}=\left\lceil\frac{s-D_{\mathrm{S}}}{D_{\mathrm{S}}+\delta}\right\rceil ; \text { and } N_{\mathrm{SW}_{\mathrm{V}}}=\left\lceil\frac{2\left(s-D_{\mathrm{S}}\right)}{\sqrt{3}\left(D_{\mathrm{S}}+\delta\right)}\right\rceil \text {. }
$$

Number of MWCNTs in Fig. 1

$$
\begin{aligned}
& N_{\mathrm{MW}_{\mathrm{h}}}=\left\lfloor\frac{w-D_{\mathrm{MW}_{\max }}}{D_{\mathrm{MW}_{\max }}+\delta}\right\rfloor \\
& N_{\mathrm{MW}_{\mathrm{v}}}=\left\lfloor\frac{h-D_{\mathrm{MW}_{\max }}}{D_{\mathrm{MW}_{\max }}+\delta}\right\rfloor
\end{aligned}
$$


where, $\lfloor X\rfloor$ and $\lceil X\rceil$ signifies that rounds each element of $X$ to the nearest integer less than or equal to that element and more than or equal to that element respectively.

Number of smaller square in Fig. 1

$$
N_{\mathrm{Sq}}=\left(N_{\mathrm{MW}_{\mathrm{h}}}-1\right)\left(N_{\mathrm{MW}_{\mathrm{v}}}-1\right)
$$

Total number of SWCNTs in the bundle

$$
N_{\mathrm{SW}}=N_{\mathrm{Sq}} N_{\mathrm{SW}}
$$

\section{Improved Mathematical Models}

After obtaining the total number of CNTs (both MWCNT and SWCNT) from the previous section, we are going to develop and extract the diameter controlled $R L C$ elements for the composite bundle of dimorphic CNTs at different technology nodes.

\subsection{Resistance in Composite CNT Bundle}

The mathematical approach to determine the equivalent resistance of the composite bundle is the obtainment of the resistance components for isolated CNTs and eventually total resistance of the bundle. To pursue the calculation, we will consider the ESC model where the resistance of the shells of MWCNTs are in parallel and adjacent CNTs are also in parallel [20]. According to [21], the quantum resistance of SWCNT can be estimated using the conductance $G=\left(\frac{2 e^{2}}{h}\right) M T$ where, $e$ is electron charge with the value of $1.62 \times 10^{-19} \mathrm{C}$

$$
R_{q}=\frac{h}{4 e^{2}} \approx 6.45 \mathrm{k} \Omega
$$

On the other hand, in case of single wall nanotube length $(l)$ exceeding the mean free path of electrons $\left(\lambda_{S W C N T}\right)$, another resistance comes up along with the former one owing to scattering which can be computed from (11).

$$
R_{S}=\frac{\hbar}{4 e^{2}}\left(\frac{1}{\lambda_{\mathrm{SWCNT}}}\right)
$$

Finally, the total resistance, emerged from previous two components of the resistance, for isolated SWCNT is denoted by (12).

$$
R_{\mathrm{SWCNT}}= \begin{cases}R_{\mathrm{C}}+R_{\mathrm{q}} & \text { if } l<<\lambda_{\mathrm{CNT}} \\ R_{\mathrm{C}}+R_{\mathrm{q}}+l R_{\mathrm{S}} & \text { if } l \geq \lambda_{\mathrm{CNT}}\end{cases}
$$

The lump resistance $\left(R_{\text {lump }}\right)$, having the quantum or intrinsic resistance from (10), engendered by the quantum detainment of electrons in a nano-wire and imperfect metal-nanotube contact resistance $\left(R_{m c}\right)$, may vary from few to several hundreds of kilo-ohms based on the fabrication process [9,12,22]. The lump resistance for different isolated MWCNTs of the proposed bundle configuration using (13), acquired from $[4,9,19,20,22,23]$.

$$
R_{\text {lump }}=\left[\sum_{i=1}^{n}\left(\frac{R_{\mathrm{q}}}{n_{\mathrm{i}}}+R_{\mathrm{mc}}\right)^{-1}\right]^{-1}
$$

The per unit length (p.u.l) scattering resistance $\left(R_{S}\right)$ emerges for length of the nanowire surpassing the effective mean free path of the electron [20]. The scattering resistance $\left(R_{S}\right)$ for different isolated MWCNTs of the proposed bundle configuration is estimated from (14) based on $[4,9,22]$. 


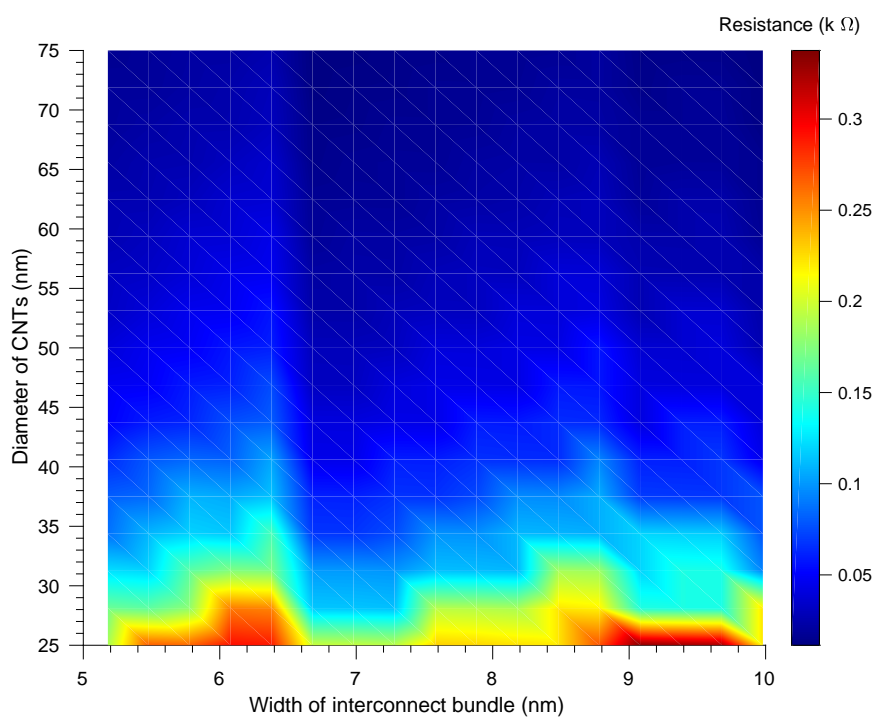

Figure 2. Synchronal variation of resistance of the squarely packed bundle of composite CNTs with altering the width of the bundle and the diameter of CNTs. Here diameter of the MWCNTs is inscribed as a function of ' $a$ ' and the diameter of SWCNTs is taken as constant value of $1 \mathrm{~nm}$. The length of the interconnect and aspect ratio are considered as $100 \mu \mathrm{m}$ and 2 respectively.

$$
R_{\mathrm{s}}=\sum_{i=1}^{n} \frac{R_{\mathrm{q}}}{n_{\mathrm{i}} \lambda_{\mathrm{i}}}
$$

The equivalent resistance of the bundle including both MWCNT and SWCNT can be recokoned using (15).

$$
R_{\text {bundle }}=\left[\left(\frac{R_{\mathrm{q}}}{N_{\mathrm{MW}}}+l \frac{R_{\mathrm{s}}}{N_{\mathrm{MW}}}\right)^{-1}+\frac{R_{\mathrm{SW}}}{N_{\mathrm{SW}}}\right]^{-1}
$$

The characteristics of resistance $(R)$ depends on both the width of the interconnect wire based on the technology node and the diameter of the used CNTs in the bundle. The simultaneous impact of both factors is observed in Figure 2. It is obvious that the lowest resistance for the bundle can be attained when both width of the bundle and the diameter of CNTs are more. Since it is taken into account that all the CNTs in the bundle are in parallel with each other, the increased number of CNTs can be obtained by increasing the width in a given space of the bundle can reduce the resistance significantly. Moreover, the increased diameter of the MWCNTs increases the number of shells which are also in parallel to each other.

\subsection{Inductance in Composite CNT Bundle}

To determine the overall inductance for our proposed configuration of the composite bundle, firstly we will calculate the inductance for isolated SWCNT and then for isolated MWCNT, finally the equivalent inductance for the entire bundle will be demonstrated as given in (21). The inductance of SWCNT consists of two components which are denoted as kinetic inductance $\left(L_{\mathrm{k}}\right)$ and magnetic inductance $\left(L_{\mathrm{m}}\right)$. Considering the ballistic conduction for a 1-D conductor, the kinetic inductance can be approximated by (16).

$$
L_{\mathrm{k}}=\frac{h}{2 e^{2} v_{F}}
$$




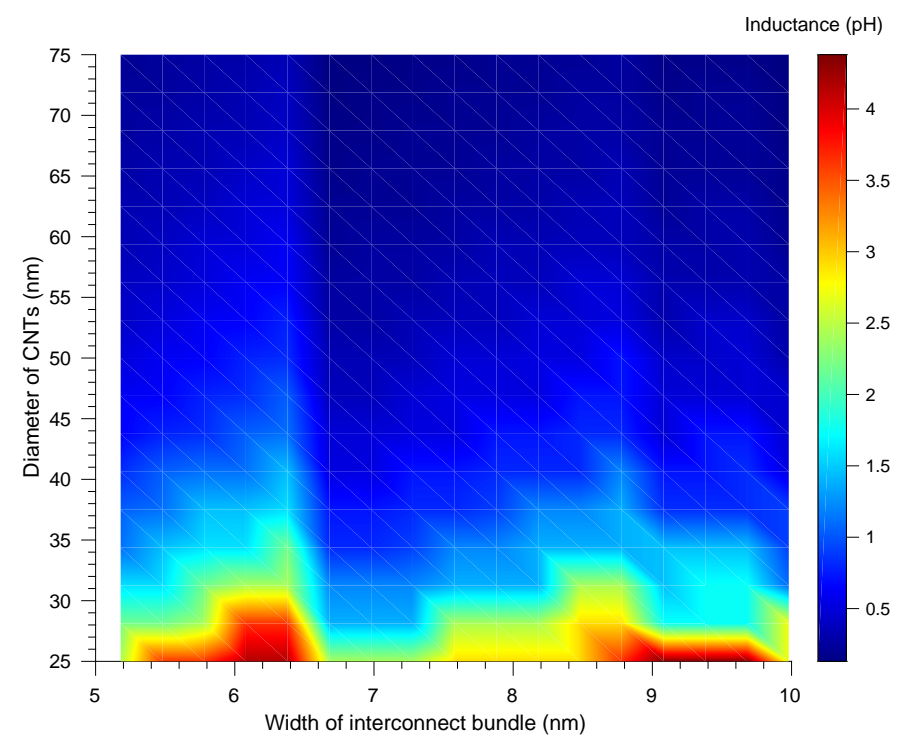

Figure 3. Concurrent extraction of inductance of the squarely packed bundle of composite CNTs with varying the width of the bundle and the diameter of CNTs. The diameter of the MWCNTs is yielded as a function of ' $a$ ' and the diameter of SWCNTs is presumed as constant value of $1 \mathrm{~nm}$. The length of the interconnect and aspect ratio are considered as $100^{-} \mathrm{m}$ and 2 respectively.

where, $v_{\mathrm{F}}$ is Fermi velocity of electron with the value of approximately $8 \times 10^{5} \mathrm{~ms}^{-1}$. Since, $L_{\mathrm{k}}$ is the function of some constant values, the approximate per unit length (p.u.l.) value is $16 \mathrm{nH} /{ }^{-} \mathrm{m} \mathrm{[24]}$. On the other hand, the stored energy of carriers in magnetic field engenders magnetic inductance, approximated in (17), in SWCNT [24].

$$
L_{\mathrm{m}}=\frac{\mu}{2 \pi} \ln \left(\frac{y}{d}\right)
$$

Now, in case of MWCNT, the magnetic inductance comes from is expressed as (20) for $i^{\text {th }}$ shell.

$$
L_{\mathrm{m}_{\mathrm{MW}}}(i)=\frac{\mu_{0}}{2 \pi} \cosh \left(\frac{2 h}{D_{M W}(i)}\right),\{i \in \mathbb{N}: 1 \leq i \leq n\}
$$

Kinetic and magnetic inductance of the isolated MWCNT in the proposed bundle is calculated using (19) and (20) respectively.

$$
\begin{gathered}
L_{\mathrm{k}_{\mathrm{MW}}}(i)=\sum_{i=1}^{n} \frac{L_{k}}{2 N_{\frac{\text { channel }}{\text { shell }_{i}}}} \\
L_{\mathrm{m}_{\mathrm{MW}}}(i)=\frac{\mu_{0}}{2 \pi} \cosh \left(\frac{2 h}{D_{M W}(i)}\right),\{i \in \mathbb{N}: 1 \leq i \leq n\}
\end{gathered}
$$

Finally, the overall equivalent inductance of the bundle is estimated in (21) which indicates that kinetic inductance component of SWCNT exists when the length of the interconnect wire exceeds the electron mean free path.

$$
L_{\text {bundle }}= \begin{cases}\left(\frac{N_{\mathrm{MW}}}{L_{\mathrm{m}_{\mathrm{MW}}+L_{\mathrm{MW}}}}+\frac{N_{\mathrm{SW}_{\Sigma}}}{L_{\mathrm{m}_{\mathrm{SW}}}+L_{\mathrm{k}_{\mathrm{SW}}}}\right)^{-1} & \text { if } l \leq \lambda_{\mathrm{CNT}} ; \\ \left(\frac{N_{\mathrm{MW}}}{L_{\mathrm{m}_{\mathrm{MW}}+L_{\mathrm{MW}}}}+\frac{N_{\mathrm{SW}}}{L_{\mathrm{m}_{\mathrm{SW}}}}\right)^{-1} & \text { if } l>\lambda_{\mathrm{CNT}} ;\end{cases}
$$

Inductance of the bundle also exhibits the same phenomena as the resistance does. The behavioral change of the inductance of the bundle with the width and diameter of 
the CNTs in the bundle is depicted in Figure 3. Based on the attained diameter, we are going to estimate the delay of the bundle in Section 4.

\subsection{Capacitance in Composite CNT Bundle}

The p. u. 1. quantum capacitance for a CNT is estimated in (22) by taking the analogy of the required energy to enclose an extra electron at an acquirable quantum state level beyond the Fermi energy level and effective capacitance. This capacitance comes into notification due to the quantum electrostatic energy stored in the nanotube during carrying the current [24].

$$
\mathrm{C}_{\mathrm{q}}=\frac{2 e^{2}}{h v_{\mathrm{F}}} \approx 0.1 \mathrm{fF} /{ }^{-} \mathrm{m}
$$

It is already mentioned to estimate the inductance for a isolated SWCNT that SWCNT has four conducting channels and these channels form a parallel combination [24]. As a result, the equivalent effective quantum capacitance of a isolated SWCNT can be approximated in (23).

$$
\mathrm{C}_{\mathrm{SW}}=4 \mathrm{C}_{\mathrm{q}} \approx 0.4 \mathrm{fF} /{ }^{-} \mathrm{m}
$$

The electrostatic capacitance, expressed in (24) is calculated in [24] by considering the SWCNT as a thin wire with diameter $D_{\text {SW }}$ putting at a distance of ' $y$ ' away from the ground.

$$
C_{\mathrm{e}}^{\mathrm{SWCNT}}=\frac{2 \pi \epsilon_{0} \epsilon_{r}}{\cosh ^{-1}\left(\frac{y}{D_{\mathrm{SW}}}\right)}
$$

Now the capacitance for isolated MWCNT is calculated using the recursive model. It is recommended in [6] that the quantum capacitance of each shell is mandatory to get determined before estimating the effective capacitance of a single MWCNT. The quantum capacitance is basically the estimation of the finite density of electronic states of quantum wire [20].

$$
C_{\mathrm{q}}=\frac{4 e^{2}}{\hbar v_{\mathrm{F}}} \sum_{i=1}^{n} N_{\mathrm{c}}(i)
$$

According to ESC model of MWCNT, it can be inferred from [23] that a shell-to-shell mutual capacitance between two adjacent shells of MWCNT.

$$
C_{\mathrm{s}}(i+1, i)=\frac{2 \pi \epsilon_{0} \epsilon_{\mathrm{r}}}{\ln \left(\frac{D_{\mathrm{i}}+2 \delta}{D_{\mathrm{i}}}\right)},\{i \in \mathbb{N}: 1 \leq i \leq n\}
$$

At first, in case of outermost shell, the equivalent capacitance, expressed in (27) represents only the quantum capacitance of that shell. As much as we go toward the inner shell, the quantum capacitance of that particular shell makes a parallel combination with the equivalent capacitance $\left(C_{\mathrm{q}-\mathrm{s}}\right)$ as shown in (29), a series combination of the capacitance of any shell and the mutual capacitance between that shell and previous shell obtained in (28), and go on until reach the innermost shell.

$$
\begin{gathered}
C_{\mathrm{ESC}}(1)=C_{\mathrm{q}}(1) \\
C_{\mathrm{q}-\mathrm{s}}(i-1)=\left(\frac{1}{C_{\mathrm{ESC}}(i-1)}+\frac{1}{C_{\mathrm{s}}(i+1, i)}\right)^{-1} \text { where, }\{i \in \mathbb{N}: 2 \leq i \leq n\} \\
C_{\mathrm{ESC}}(i)=C_{\mathrm{q}}(i)+C_{\mathrm{q}-\mathrm{s}}(i-1) \text { where, }\{i \in \mathbb{N}: 2 \leq i \leq n\}
\end{gathered}
$$




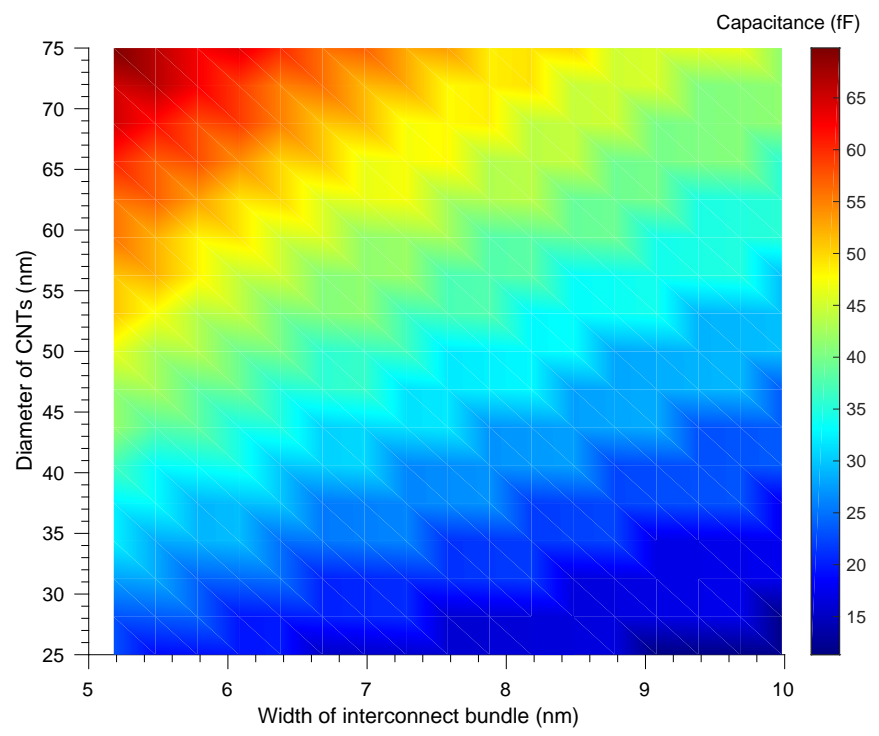

Figure 4. Contemporaneous denouement of capacitance of the squarely packed bundle of composite CNTs with varying the width of the bundle and the diameter of CNTs. The diameter of the MWCNTs is inferred as a function of ' $a$ ' and the diameter of SWCNTs is taken as constant value of $1 \mathrm{~nm}$. The length of the interconnect and aspect ratio are considered as $100^{-} \mathrm{m}$ and 2 respectively.

The electrostatic capacitance demonstrated the potential difference between the $\mathrm{CNT}$ over the ground plane and ground [20]. The p. u. 1. electrostatic capacitance can be approximated in (30).

$$
C_{\mathrm{E}}=\frac{2 \pi \epsilon_{0} \epsilon_{\mathrm{r}}}{\ln \left(\frac{D_{\mathrm{i}}+2 \delta}{D_{\mathrm{i}}}\right)}
$$

The conglomerate capacitance of the proposed composite bundle is obtained in (31) by considering the overall effect of SWCNT and MWCNT in the bundle. To estimate this, we considered the effect of electrostatic capacitance of MWCNTs over the ground plane on the effective capacitance in series.

$$
C_{\text {bundle }}=\frac{N_{\mathrm{MW}} C_{\mathrm{E}}\left(N_{\mathrm{MW}} C_{\mathrm{ESC}_{\mathrm{MW}}}+N_{\mathrm{SW}_{\Sigma}} C_{\mathrm{SW}}\right)}{N_{\mathrm{MW}} C_{\mathrm{ESC}_{\mathrm{MW}}}+N_{\mathrm{SW}_{\Sigma}} C_{\mathrm{SW}}+N_{\mathrm{MW}_{\mathrm{H}}} C_{\mathrm{E}}}
$$

Unlike the resistance and inductance, capacitance shows descending behavior with lower diameter of CNTs in the bundle. We can also notice from Figure 4 that capacitance decreases further in higher technology node. The reason behind this phenomenon is that the capacitance components arisen from parallel CNTs magnify the equivalent capacitance in the bundle.

\section{Simulation Results}

This section illustrates the performance comparison of squarely packed bundle of dimorphic MWCNTs and that of composite CNTs to exploit the feature of using composite CNTs in the interconnect bundle. To observe the performance in terms of propagation delay, we simulate the Kahng's model, obtained from [13] [8], using the extracted equivalent value of RLC and optimized dimension of CNTs in Section 3. Subsequently, we assess the performance of the proposed configuration in terms of crosstalk delay, excerpted from [4], using the optimized size of particular CNTs and extracted $R L C$ in Section 3 and number of CNTs in Section 2. 


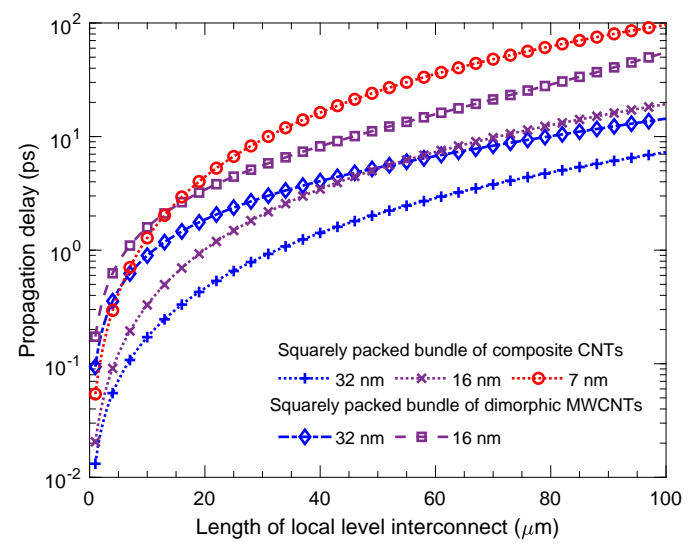

Figure 5. Comparison of delay performance of Squarely packed bundle of composite CNTs and that of dimorphic MWCNTs for local level interconnect length. Earlier one doesn't seem suitable for $7 \mathrm{~nm}$ technology node due to the lack of space for MWCNTs with both sizes. The size and number of accommodated CNTs for different technology nodes are mentioned in Table 1.

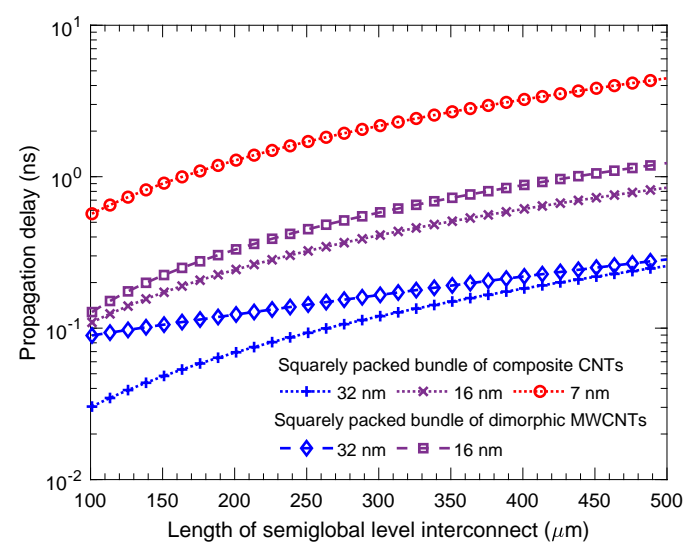

Figure 6. Illustration of comparative delay performance of Squarely packed bundle of composite CNTs and that of dimorphic MWCNTs for semiglobal level interconnect length. It doesn't include the delay performance of Squarely packed bundle of dimorphic MWCNTs for $7 \mathrm{~nm}$ technology node due to the lack of space for MWCNTs with both sizes. The size and number of accommodated CNTs for different technology nodes are mentioned in Table 1.

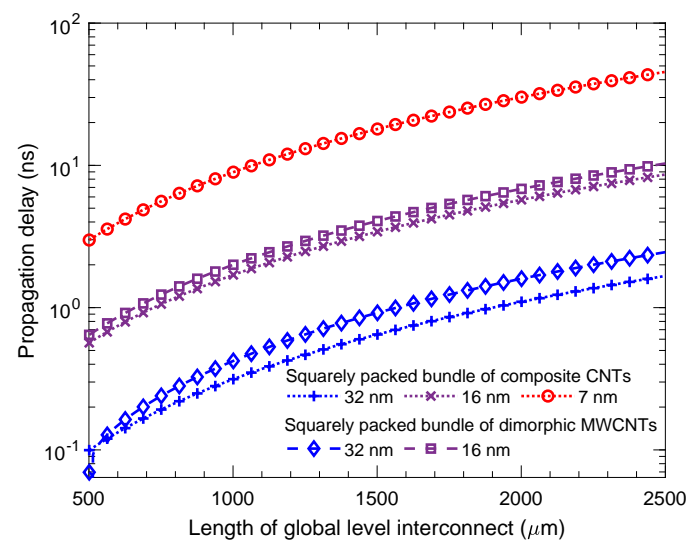

Figure 7. Demonstration of comparison between the propagation delay performance of Squarely packed bundle of composite CNTs and that of dimorphic MWCNTs for global level interconnect length. It doesn't include the delay performance of Squarely packed bundle of dimorphic MWCNTs for $7 \mathrm{~nm}$ technology node due to the lack of space for MWCNTs with both sizes. The size and number of accommodated CNTs for different technology nodes are mentioned in Table 1. 
Table 1. Diameter and number of pertinent CNTs accommodated in the bundle during simulation to obtain propagation delay and crosstalk delay.

\begin{tabular}{|c|c|c|c|c|c|c|c|c|c|}
\hline \multirow{2}{*}{$\begin{array}{l}\text { Interconnect } \\
\text { length }(\mu \mathrm{m})\end{array}$} & \multirow{2}{*}{$\begin{array}{l}\text { Technology } \\
\text { node (nm) }\end{array}$} & \multicolumn{4}{|c|}{ Squarely Packed Dimorphic } & \multicolumn{4}{|c|}{ Squarely Packed Composite } \\
\hline & & $\begin{array}{c}D_{\mathrm{MW}_{\max }}^{L} \\
(\mathrm{~nm})\end{array}$ & $N_{\mathrm{MW}}^{L}$ & $\begin{array}{c}D_{\mathrm{MW}_{\max }^{S}}^{S} \\
(\mathrm{~nm})\end{array}$ & $N_{\mathrm{MW}}^{S}$ & $\begin{array}{c}D_{\mathrm{MW}_{\max }} \\
\text { (nm) }\end{array}$ & $N_{\mathrm{MW}}$ & $\begin{array}{l}D_{\mathrm{SW}} \\
(\mathrm{nm})\end{array}$ & $N_{S W_{\Sigma}}$ \\
\hline \multirow{3}{*}{$\begin{array}{c}\text { Local } \\
(0-100)\end{array}$} & $32 \mathrm{~nm}$ & 10 & 32 & 4.31 & 21 & 10 & 21 & 1 & 96 \\
\hline & $16 \mathrm{~nm}$ & 8.5 & 8 & 3.72 & 3 & 8.5 & 8 & 1 & 15 \\
\hline & $7 \mathrm{~nm}$ & - & - & - & - & 4.5 & 3 & 1 & 8 \\
\hline \multirow{3}{*}{$\begin{array}{c}\text { Semiglobal } \\
(101-500)\end{array}$} & $32 \mathrm{~nm}$ & 10 & 32 & 4.31 & 21 & 10 & 21 & 1 & 96 \\
\hline & $16 \mathrm{~nm}$ & 8.5 & 8 & 3.72 & 3 & 8.5 & 8 & 1 & 15 \\
\hline & $7 \mathrm{~nm}$ & - & - & - & - & 4.5 & 3 & 1 & 8 \\
\hline \multirow{3}{*}{$\begin{array}{c}\text { Global } \\
(501-2500)\end{array}$} & $32 \mathrm{~nm}$ & 10 & 48 & 4.31 & 33 & 10 & 33 & 1 & 160 \\
\hline & $16 \mathrm{~nm}$ & 8.5 & 14 & 3.72 & 6 & 8.5 & 12 & 1 & 25 \\
\hline & $7 \mathrm{~nm}$ & - & - & - & - & 4.5 & 12 & 1 & 5 \\
\hline
\end{tabular}

It is demonstrable from Figure 5-7 that our proposed configuration yields lower propagation delay than the preceding configuration from [16] does. It is conspicuous from Table 1 that we can increase the number of CNTs without distorting the overall configuration of squarely packed bundle using the proposed approach. As a consequence, the resistance and inductance decreases while the capacitance increases for any specific technology node. Finally, the overall impact decreases the propagation delay for the squarely packed bundle of composite CNTs which is represented in the Figure 5, Figure 6 and Figure 7 for local, semiglobal and global level respectively. However, the preceding squarely packed bundle configuration doesn't seem suitable for $7 \mathrm{~nm}$ technology node because of unavailability of space to accommodate the CNTs with various sizes. Hence, the simulation illustrations don't include the delay of squarely packed bundle of dimorphic MWCNTs for $7 \mathrm{~nm}$ technology. The remarkable point to add here that our proposed configuration is perfectly adaptable for technology shrinkage along with performance enhancement for all technology nodes i.e. $32 \mathrm{~nm}, 16 \mathrm{~nm}$ and $7 \mathrm{~nm}$.

The crosstalk delay basically arises from the capacitance formed between the CNTs from different bundles while it is considered that all CNTs in the bundles are in parallel [4]. The inter bundle capacitance, function of spacing between the the centers of two adjacent CNTs, average diameter of the adjacent CNTs and relative permittivity based on the level of interconnect length, is estimated by (32) where $D_{\mathrm{MW}_{\max }}$ is used as diameter because we placed MWCNT on the edge of the bundle to reduce the overall crosstalk impact by following $[6,9]$.

$$
C_{\mathrm{cm}_{\mathrm{ESC}}}=\frac{\pi \epsilon_{0} \epsilon_{\mathrm{r}}}{\cosh ^{-1}\left(\frac{S_{\mathrm{p}}}{D_{\mathrm{MW} \text { max }}}\right)} N_{\mathrm{MW}}
$$

Eventually, the crosstalk performance of our proposed configuration is depicted in Figure 8, Figure 9 and Figure 10 for local, semiglobal and global level interconnect by comparing with the preceding configuration from [16]. It is noticeable from Figure 9 that crosstalk performance of the proposed configuration in a substantial manner for both $32 \mathrm{~nm}$ and $16 \mathrm{~nm}$ technology nodes. On the other hand, Figure 9 illustrates that the crosstalk performance betterment is more in $32 \mathrm{~nm}$ than in $16 \mathrm{~nm}$ technology node. In case of global level interconnect, the amount of crosstalk delay performance enhancement of our proposed configuration for both $32 \mathrm{~nm}$ and $16 \mathrm{~nm}$ technology nodes is almost same as it is illustrated in Figure 10. It is also demonstrable that our proposed configuration is appropriate for $7 \mathrm{~nm}$ technology node. 


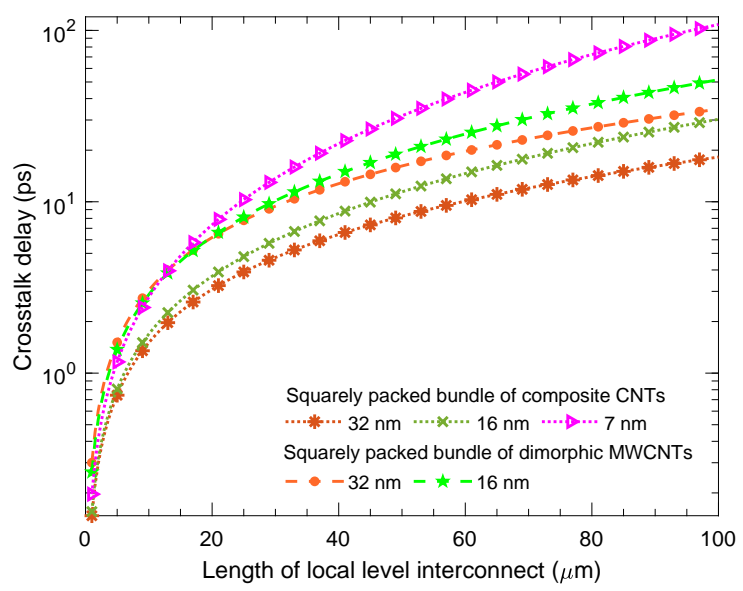

Figure 8. Comparative exhibition of crosstalk delay of proposed and previously developed bundle configuration for different technology nodes at local level interconnect length. The dimension of used CNTs are same as used in the simulation for obtaining propagation delay at local level.

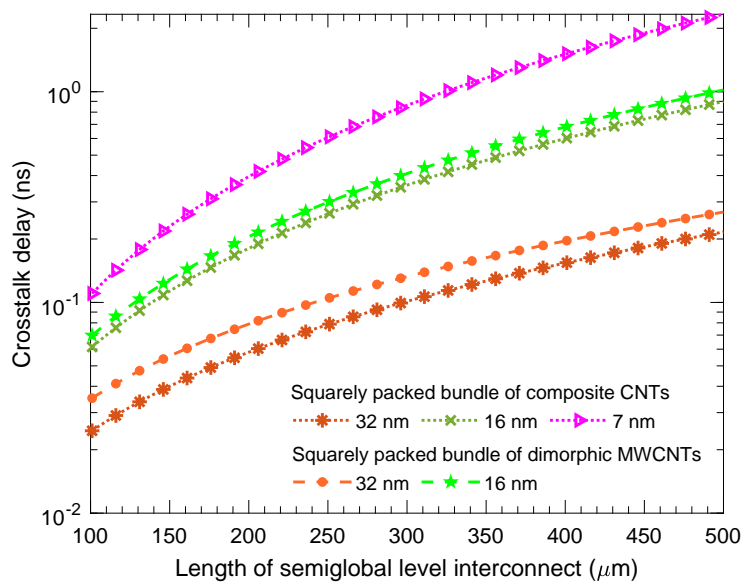

Figure 9. Comparative analysis of crosstalk delay of proposed and previously developed bundle configuration for different technology nodes at semiglobal level interconnect length. The dimension of used CNTs are same as used in the simulation for obtaining propagation delay at semiglobal level.

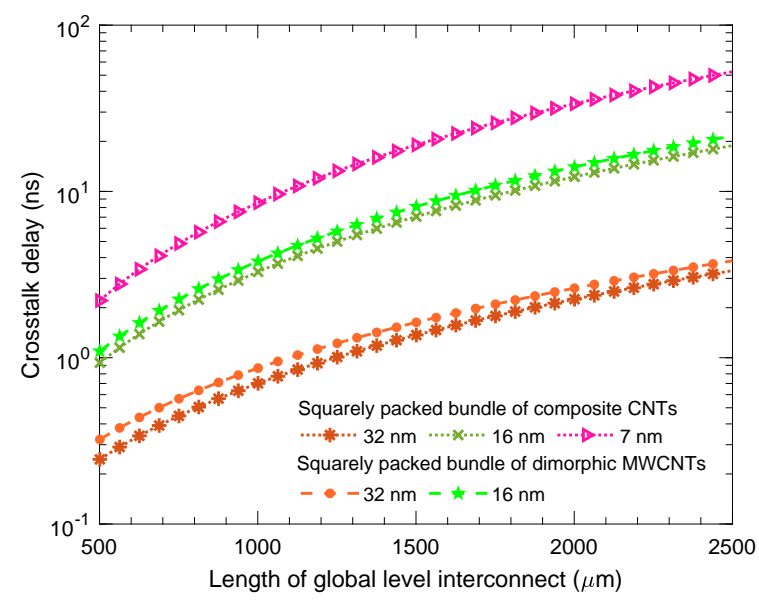

Figure 10. Comparative illustration of crosstalk delay of proposed and previously developed bundle configuration for different technology nodes at global level interconnect length. The diameter and number of used CNTs are same as used in the simulation for obtaining propagation delay at global level. 


\section{Conclusion}

A modified configuration of the squarely packed bundle CNTs is presented by following the previously developed configuration in [16]. By proposing this configuration, applicability of both MWCNT and SWCNT simultaneously for scaled interconnect in future VLSI integrated circuit is analyzed abstractly. The obtained propagation delay and crosstalk delay performance using $R L C$ model and delay model exhibits the transcendence of squarely packed bundle of composite CNTs for local, semiglobal and global level interconnect at $32 \mathrm{~nm}, 16 \mathrm{~nm}$ and $7 \mathrm{~nm}$ technology nodes.

In this approach, CNTs with only two different sizes are used. In the upcoming endeavor, our intention is to advance the work by adding the CNTs with various sizes to make the configuration more convenient in terms of fabrication process.

\section{References}

1. Li, H.; Yin, W.Y.; Banerjee, K.; Mao, J.F. Circuit modeling and performance analysis of multi-walled carbon nanotube interconnects. IEEE Transactions on electron devices 2008, 55, 1328-1337.

2. Kabir, M.S. Controlled growth of a nanostructure on a substrate, and electron emission devices based on the same, 2011. US Patent 7,977,761.

3. Liew, K.; Wong, C.; He, X.; Tan, M. Thermal stability of single and multi-walled carbon nanotubes. Physical Review B 2005, 71,075424 .

4. Kaushik, B.K.; Majumder, M.K. Carbon nanotube based VLSI interconnects: Analysis and design; Springer, 2015.

5. Pu, S.; Yin, W.; Mao, J.; Liu, Q.H. Crosstalk Prediction of Single- and Double-Walled Carbon-Nanotube (SWCNT/DWCNT) Bundle Interconnects. IEEE Transactions on Electron Devices 2009, 56, 560-568.

6. Subash, S.; Kolar, J.; Chowdhury, M.H. A new spatially rearranged bundle of mixed carbon nanotubes as VLSI interconnection. IEEE Transactions on Nanotechnology 2011, 12, 3-12.

7. Kim, S.; Kulkarni, D.D.; Rykaczewski, K.; Henry, M.; Tsukruk, V.V.; Fedorov, A.G. Fabrication of an ultralow-resistance ohmic contact to MWCNT-metal interconnect using graphitic carbon by electron beam-induced deposition (EBID). IEEE transactions on nanotechnology 2012, 11, 1223-1230.

8. Amin, A.B.; Ullah, M.S. Mathematical Framework of Tetramorphic MWCNT Configuration for VLSI Interconnect. IEEE Transactions on Nanotechnology 2020, 19, 749-759. doi:10.1109/TNANO.2020.3026609.

9. Majumder, M.K.; Kaushik, B.K.; Manhas, S.K. Analysis of delay and dynamic crosstalk in bundled carbon nanotube interconnects. IEEE transactions on electromagnetic compatibility 2014, 56, 1666-1673.

10. Rai, M.K.; Garg, H.; Kaushik, B. Temperature-dependent modeling and crosstalk analysis in mixed carbon nanotube bundle interconnects. Journal of Electronic Materials 2017, 46, 5324-5337.

11. Sharma, M.; Rai, M.K.; Khanna, R. Temperature-dependent crosstalk and frequency spectrum analyses in adjacent interconnects of a mixed CNT bundle. Journal of Computational Electronics 2020, 19, 177-190.

12. Sandha, K.S.; Thakur, A. Comparative Analysis of Mixed CNTs and MWCNTs as VLSI Interconnects for Deep Sub-micron Technology Nodes. Journal of Electronic Materials 2019, 48, 2543-2554.

13. Kahng, A.B.; Muddu, S. An analytical delay model for RLC interconnects. IEEE Transactions on Computer-Aided Design of Integrated Circuits and Systems 1997, 16, 1507-1514.

14. Ullah, M.S.; Chowdhury, M.H. Analytical models of high-speed RLC interconnect delay for complex and real poles. IEEE Transactions on Very Large Scale Integration (VLSI) Systems 2017, 25, 1831-1841.

15. Sanaullah, M.; Chowdhury, M.H. Analysis of RLC interconnect delay model using second order approximation. 2014 IEEE International Symposium on Circuits and Systems (ISCAS), 2014, pp. 2756-2759. doi:10.1109/ISCAS.2014.6865744.

16. Amin, A.B.; Ullah, M.S. Performance Analysis of Squarely Packed Dimorphic MWCNT Bundle for High Speed VLSI Interconnect. 2020 IEEE Canadian Conference on Electrical and Computer Engineering (CCECE), 2020, pp. 1-6. doi:10.1109/CCECE47787.2020.9255744.

17. Amin, A.B.; Ullah, M.S. Performance Analysis of Squarely Packed Polymorphic SWCNT Interconnect. 2019 IEEE 10th Annual Ubiquitous Computing, Electronics Mobile Communication Conference (UEMCON), 2019, pp. 1199-1203. doi:10.1109/UEMCON47517.2019.8992975.

18. Naeemi, A.; Meindl, J.D. Physical modeling of temperature coefficient of resistance for single-and multi-wall carbon nanotube interconnects. IEEE Electron Device Letters 2007, 28, 135-138.

19. Das, D.; Rahaman, H. Analysis of crosstalk in single-and multiwall carbon nanotube interconnects and its impact on gate oxide reliability. IEEE Transactions on Nanotechnology 2011, 10, 1362-1370.

20. Majumder, M.K.; Das, P.K.; Kaushik, B.K. Delay and crosstalk reliability issues in mixed MWCNT bundle interconnects. Microelectronics Reliability 2014, 54, 2570-2577.

21. Srivastava, N.; Li, H.; Kreupl, F.; Banerjee, K. On the applicability of single-walled carbon nanotubes as VLSI interconnects. IEEE Transactions on Nanotechnology 2009, 8, 542-559. 
22. Sahoo, M.; Ghosal, P.; Rahaman, H. Modeling and analysis of crosstalk induced effects in multiwalled carbon nanotube bundle interconnects: An ABCD parameter-based approach. IEEE Transactions on Nanotechnology 2015, 14, $259-274$.

23. Sarto, M.S.; Tamburrano, A. Single-conductor transmission-line model of multiwall carbon nanotubes. IEEE Transactions on Nanotechnology 2009, 9, 82-92.

24. Banerjee, K.; Srivastava, N. Are carbon nanotubes the future of VLSI interconnections? Proceedings of the $43 \mathrm{rd}$ annual Design Automation Conference, 2006, pp. 809-814. 